\title{
LA VULNERACIÓN DE LOS DERECHOS CONSTITUCIONALES DE LOS CIUDADANOS CONSUMIDORES DE CANNABIS SATIVA POR PARTE DE LA RESOLUCIÓN 002- CONSEP-CD-2015
}

\section{THE VIOLATION OF THE CONSTITUTIONAL RIGHTS OF CITIZENS WHO CONSUME CANNABIS SATIVA BY RESOLUTION 002-CONSEP-CD-2015}

\author{
Jorge Ortega Mohina, Mgtr. \\ Magíster en Derecho Constitucional (Ecuador). \\ Abogado en libre ejercicio profesional, Guayaquil Ecuador. \\ jorgemarxortega@gmail.com
}

\section{ARTÍCULO DE REFLEXIÓN}

Recibido: 2 de diciembre de 2020

Aceptado: 9 de marzo de 2021

\section{RESUMEN}

Esta investigación posee como objeto de estudio la criminalización del consumo y vulneración de los derechos constitucionales de los consumidores de cannabis sativa, la referida problemática será analizada en virtud de tres objetivos específicos y un objetivo general; los cuales se encuentran orientados a fundamentar jurídicamente la no criminalización de los consumidores de cannabis sativa, identificar los parámetros que otorgan la calidad de consumidor de cannabis sativa a un ciudadano y analizar la constitucionalidad de la resolución Nro. 002-CONSEP-CD-2015, dichos objetivos permitirán determinar la vulneración a los derechos constitucionales de los ciudadanos consumidores de cannabis sativa por parte de la referida resolución. La metodología aplicada en el presente estudio reside en los métodos analítico teórico, teórico jurídico, exegético analítico y jurídico comparando. En base a esas consideraciones, los resultados más relevantes obtenidos residen en la fundamentación jurídica respecto a la no criminalización de los consumidores de cannabis sativa y la identificación de los parámetros que otorgan la calidad de consumidor de cannabis sativa a un ciudadano en el ordenamiento jurídico ecuatoriano, además de la determinación de la incompatibilidad de la resolución 002-CONSEP-CD-2015 con el artículo 364 de la constitución debido a la vulneración de derechos que comete en contra de los consumidores de cannabis sativa.

Palabras claves: criminalización, derechos constitucionales, cannabis sativa, consumidor 


\section{ABSTRACT}

This research has as its object of study the criminalization of consumption and violation of the constitutional rights of cannabis sativa consumers. The aforementioned problem will be analyzed by virtue of three specific objectives and one general objective; which are aimed at legally substantiating the non-criminalization of cannabis sativa consumers, identifying the parameters that grant the quality of cannabis sativa consumer to a citizen and analyzing the constitutionality of resolution No. 002-CONSEP-CD-2015, These objectives will make it possible to determine the violation of the constitutional rights of citizens who consume cannabis sativa by the aforementioned resolution. The methodology applied in the present study resides in the theoretical analytical, legal theoretical, analytical exegetical and legal methods by comparing. Based on these considerations, the most relevant results obtained reside in the legal basis regarding the non-criminalization of cannabis sativa consumers and the identification of the parameters that grant the quality of consumer of cannabis sativa to a citizen in the Ecuadorian legal system , in addition to the determination of the incompatibility of resolution 002-CONSEPCD-2015 with article 364 of the constitution due to the violation of rights committed against consumers of cannabis sativa.

Keywords: criminalization, constitutional rights, cannabis sativa, consumer

\section{INTRODUCCIÓN}

El presente trabajo critica la incompatibilidad existente entre la disposición constitucional que establece a las adicciones como un problema de salud pública, la no criminalización de los consumidores y la no vulneración de sus derechos constitucionales; contra el desarrollo de los mecanismos de control del consumo de sustancias estupefacientes y psicotrópicas, específicamente a la Resolución Nro. 002-CONSEP-CD-2015 emitida por el Consejo Nacional de Control de Sustancias Estupefacientes y Psicotrópicas publicada en Registro Oficial Suplemento 628 de 16 de noviembre de 2015.

Sin duda alguna, el incremento en los índices de consumo de sustancias estupefacientes y psicotrópicas en nuestro país, han convertido a las drogas, no solo en un problema de salud pública, sino también, las ha configurado como un fenómeno socio económico que conmociona a nuestra sociedad en todos sus estratos. El presente estudio se enfocará en el consumo y tenencia de cannabis sativa, al ser esta, una de las sustancias sujetas a fiscalización con evidente circulación; y, por la legalidad de la misma dentro de ordenamientos jurídicos extranjeros, en un 
ámbito de uso medicinal y recreativo. Respecto a esta temática la Defensoría Pública del Ecuador, presentó una propuesta de reforma al Código Orgánico Integral Penal en el año 2016 la misma que se encuentra fundamentada en lo siguiente:

En Ecuador existe un estándar constitucional establecido en el artículo 364 que prohíbe criminalizar al consumidor de drogas de uso ilícito, resaltando que las adicciones son un problema de salud pública, esto es, que el fenómeno social de las drogas debe ser abordado con políticas públicas de prevención y salud y no con el Código Penal. Por ello, con la finalidad de evitar una distorsión en el sistema penal, el proyecto se encamina exclusivamente a aclarar que la tenencia o posesión de cantidades pequeñas evidencia objetivamente la existencia de consumo y no de microtráfico (Pazmiño, 2016, págs. 13-14)

El análisis en cuestión no se centrará en determinar si el consumidor posee el Derecho Constitucional al libre consumo de sustancias estupefacientes o psicotrópicas, o mucho menos a establecer opiniones subjetivas acerca de que el consumo de estas sustancias se configura en un derecho; el examen se fijará en determinar si la Resolución Nro. 002-CONSEP-CD-2015 a través de su accionar llega a violentar derechos constitucionales como la libertad, seguridad jurídica, no discriminación e igualdad, derechos de los cuales los ciudadanos consumidores de cannabis sativa son titulares al igual que cualquier otro ciudadano y que la garantía de los mismos son responsabilidad del Estado, tal como lo manifiesta nuestra constitución en el numeral uno del artículo tres.

Nuestra ley fundamental determina expresamente que, en ningún caso se permitirá la criminalización del consumo de sustancias estupefacientes y psicotrópicas; ni la vulneración de los derechos constitucionales de los consumidores. La Ley Orgánica de Prevención Integral del Fenómeno Socio Económico de las drogas manifiesta que no se podrán interferir, limitar o violar el ejercicio de los derechos de los consumidores. Asimismo, señala que no se podrá criminalizar el uso o consumo de drogas, en los términos establecidos por la ley; así como, la no discriminación o estigmatización de los consumidores.

Es cierto que se ha determinado que el consumo no puede ser criminalizado pero, para poder instaurar un control al narcotráfico y efectivizar el cumplimiento del artículo 220 de Código Orgánico Integral Penal que castiga el tráfico ilícito de sustancias sujetas a fiscalización, el Estado ecuatoriano a través de su órgano competente (CONSEP), emitió la Resolución Nro. 002CONSEP-CD-2015, la cual determina como tráfico llícito a Mínima Escala de marihuana (cannabis sativa), a la persona que porte desde $>0$ gramos hasta 20 gramos; esto se contrapone con la resolución 001-CONSEP-CD-2013, la cual no establece las tablas de cantidades de 108 
sustancias estupefacientes y psicotrópicas para sancionar el tráfico ilícito de mínima, mediana, alta y gran escala, pero si identifica que la cantidad máxima admisible de tenencia para consumo personal en el caso de marihuana (cannabis sativa) es 10 gramos.

Es en este escenario donde se identifica que un ciudadano al poseer hasta 10 gramos de cannabis sativa, adquiere la calidad de consumidor, ya que esta es la cantidad de tenencia máxima permitida para el consumo personal de acuerdo a lo dispuesto por la resolución 001CONSEP-CD-2013, pero también, al portar la cantidad referida se encontraría adecuando su conducta en el cometimiento del delito de tráfico ilícito de mínima escala de sustancias sujetas a fiscalización acorde a lo establecido por la Resolución 002-CONSEP-CD-2015.

Además, la tabla contenida en la referida resolución solo se limita a la consideración cuantitativa de la tenencia de sustancias sujetas a fiscalización para sancionar el delito de tráfico ilícito y no a identificar si el destino de la tenencia se encuentra orientado al tráfico. Entonces, es aquí donde encontramos una posible vulneración a los derechos constitucionales de los consumidores.

\section{REVISIÓN TEÓRICA}

\section{Interpretación de la disposición constitucional contenida en el artículo 364 de la Constitución de la República del Ecuador}

El presente examen fijará su estudio en la interpretación de la disposición constitucional contenida en el artículo 364 de la constitución a través de los diferentes métodos de interpretación aplicables al caso, los cuales se encuentran establecidos en la Ley Orgánica de Garantías Jurisdiccionales y Control Constitucional, conjuntamente con los métodos atribuibles y desarrollados por la doctrina.

Nuestra constitución establece en su artículo 427 que las normas constitucionales son obtenidas en base a una interpretación por el tenor literal que más se ajuste a la Constitución en su integralidad. En este punto es sustancial recalcar que el constituyente de forma errónea ha señalado la "interpretación de normas" puesto que la interpretación es una actividad del intelecto humano que recae sobre las disposiciones, es decir, sobre lo escrito en los cuerpos de leyes y consecuentemente, como resultado de la referida actividad se obtiene el nacimiento o la concepción de una norma. De la lectura del artículo 427 de la constitución se identifica que el primer método a utilizar al momento de la interpretación de una disposición es, sin lugar a dudas, la interpretación literal.

109 
La Ley Orgánica de Garantías Jurisdiccionales y Control Constitucional determina en su artículo 3 que la interpretación literal consiste en que, al existir una disposición clara, esta debe atenderse a su tenor literal. En esa línea de análisis, se identifica que el primer sentido de la interpretación literal es a través de un lenguaje común que se encuentre dotado de una compresibilidad clara y entendible a todo el auditorio social sin la necesidad de que sus integrantes deban poseer conceptos jurídicos previamente adquiridos. Entonces, utilizando el lenguaje común se interpreta que la disposición del artículo 364 de la constitución determina 3 parámetros principales:

\section{Las adicciones son un problema de salud pública}

Por la palabra "adiciones" se entiende que adicción es una dependencia a una sustancia o actividad que traen como consecuencias resultados negativos a la salud física y mental de un individuo. Respecto a "problema" se entiende que esta palabra describe a un hecho, circunstancia o un conjunto de los mismos, que representa una dificultad para obtener un fin. Adicionalmente, la disposición constitucional dota con un adjetivo de pertenencia a la palabra "problema" puesto que, le atribuye una cualidad al determinar que no es un problema común, sino un problema de "salud pública".

Entonces, "salud pública" es un término que no es comúnmente usado, sin embargo, se dilucida que la "salud pública" se concentra en prevenir las enfermedades, no curarlas; conservar la salud, prolongar la conservación de la vida y mejorar la condición física de la especie humana, orientar la higiene para que desaparezcan las enfermedades evitables y lo más importante es que esta actividad no recae únicamente en el ciudadano, sino que es una responsabilidad primordial del Estado.

El Estado es responsable de la información, prevención, control de consumo, tratamiento y rehabilitación de los consumidores ocasionales, habituales y problemáticos de alcohol, tabaco y sustancias estupefaciente y psicotrópicas

Debido a los diferentes significados atribuibles a la palabra "Estado", en este particular caso recurriré al empleo del lenguaje técnico-jurídico elemental para poder entender el significado de "Estado", y es así, que se identifica que un Estado es una forma de organización política, dotada de un poder soberano e independiente con reconocimiento a nivel internacional, el cual posee órganos de gobiernos propios y que se asienta en un determinado territorio habitado principalmente por personas, es decir, un país soberano como lo es Ecuador.

110 
Siguiendo con la deconstrucción del enunciado, se aprecia que la disposición constitucional contenida en el artículo 364 de la constitución le atribuye responsabilidades al Estado ecuatoriano respecto a la problemática del consumo de sustancias sujetas a fiscalización, tales como: "información", actividad de comunicar los efectos y consecuencias del uso de sustancias sujetas a fiscalización; "prevención", preparación anticipada para evitar el riesgo que trae consigo el abuso de este tipo de sustancias; "control", es la inspección e intervención del Estado ecuatoriano respecto al uso de sustancias sujetas a fiscalización, así como la publicidad de las mismas; "consumo", utilización de las referidas sustancias; "tratamiento", conjunto de medios que se emplean para aliviar o curar una enfermedad; "rehabilitación", conjunto de métodos por el cual se busca obtener la recuperación de una actividad o función perdida o disminuida a causa de una enfermedad o traumatismo; "consumidores", personas que pagan por un producto o los usuarios finales del mismo, puntualizando que el pago y el uso son actividades de índole distintas; "consumidores ocasionales" personas que consumen o adquieren sustancias sujetas a fiscalización en momentos aleatorios o indeterminados; "consumidores habituales" personas que consumen o adquieren sustancias sujetas a fiscalización con continuidad o por hábito; "consumidores problemáticos" personas que consumen sustancias sujetas a fiscalización y como consecuencia de dicha actividad causan problemas o inconvenientes a sí mismos o a la comunidad.

En el presente caso no me referiré al entendimiento de las palabras "alcohol" o "tabaco" puesto que, las mismas al ser parte constitutiva de nuestra cultura coloquial son totalmente comprensibles, pero si me referiré a "estupefacientes" como sustancias que alteran la sensibilidad y pueden producir efectos estimulantes, deprimentes, narcóticos, o alucinógenos y cuyo uso continuado puede causar adicciones, y; "psicotrópicas", sustancias psicoactivas que producen efectos por lo general intensos, hasta el punto de causar cambios profundos de personalidad.

\section{La criminalización de los consumidores ni se vulnerarán sus derechos constitucionales}

Continuando con la utilización del lenguaje común y técnico-jurídico para el entendimiento del presente enunciado, se identifica que, "ningún" es un vocablo por el cual se determina inexistencia; "caso" significa suceso, acontecimiento u ocasión; "permitir", acción por la cual es posible realizar una actividad; "criminalización" es la materialización de atribuir el carácter de criminal o una conducta delictiva a un individuo; "vulnerar", se entiende como la acción de transgredir, quebrantar, violar, dañar o perjudicar, y; "derechos constitucionales" son principios fundamentales exigibles por sus titulares (derecho a la libertad, no discriminación, igualdad), los 111

RES NON VERBA, ISSN impreso: 1390-6968 | e-ISSN: 2661-6769, Vol.11, No. 1, abril de 2021 
cuales no solo se encuentran determinados en la constitución de un Estado, sino que estos han adquirido un efecto de irradiación sobre todo el ordenamiento jurídico, lo que ha traído consigo una "omnipresencia" de los derechos constitucionales.

Al referirnos a los derechos constitucionales no se debe limitar únicamente a la consideración de que los mismos son la positivización de los derechos fundamentales en la Ley Fundamental, sino también a entender que los derechos constitucionales se encuentran vinculados directamente al principio de proporcionalidad, por lo que, la aplicabilidad de este principio admite que los derechos constitucionales pueden categorizarse como principios o mandatos de optimización.

Por las consideraciones expuestas, bajo una cabal interpretación literal de la disposición contenida en el artículo 364 de la constitución, se demuestra que la norma constitucional producto de esta interpretación determina que las adicciones son afecciones a la salud, consecuentemente esto genera un problema a las personas que la poseen, pero que este problema de salud no es una dificultad de carácter exclusiva al individuo, sino que al ser las adicciones catalogadas como un problema de salud pública, la responsabilidad de combatir las mismas y solucionarlas recaen sobre el Estado, ya que su deber más alto es garantizar a los ciudadanos los derechos que emanan de la constitución.

Además de aquello, esta disposición constitucional ha dotado de actividades específicas al Estado para combatir este problema de salud pública, y estas son la información, la prevención, el control, tratamiento y rehabilitación de las personas consumidoras independientemente de la frecuencia o consecuencia de su consumo (ocasionales, habituales o problemáticos). Finalmente, el constituyente en su correcta concepción de que el mayor garantista de derechos es el Estado, ha determinado contundentemente que, en ningún caso, situación o escenario, las personas consumidoras de alcohol, tabaco, estupefacientes y psicotrópicos podrán ser criminalizadas, es decir, que la acción de consumir las referidas sustancias, en ningún caso podrá ser considerada como una conducta que se adecue al cometimiento de un delito, por lo tanto, un consumidor no podrá ser sujeto de una sanción penal que trae como resultado su privación de libertad (derecho constitucional), ni mucho menos por su acción de consumir podrá ser sujeto a violaciones de sus derechos constitucionales como los son la igualdad, no discriminación, salud, educación y todos los demás derechos que emanen de nuestra ley fundamental.

Las críticas que recaen sobre la interpretación literal se fundamentan en la simplicidad de este tipo de interpretación y a su no compatibilidad absoluta, debido a que las diversidades de la naturaleza humana a través de sus distintas manifestaciones en casos concretos devienen en 112 
particularidades que dificultan que una sola norma sea la única para poder tratar las múltiples variables, por aquella razón, nuestra constitución en el artículo 427 determina que las disposiciones deben ser interpretadas en su tenor literal pero siempre que esta interpretación literal sea la que más se adecue a la constitución en su integralidad, concordantemente a esto, la Ley Orgánica de Garantías Jurisdiccionales de Control Constitucional determina que para poder obtener un resultado justo, se podrán accionar otros métodos de interpretación sin perjuicio de la utilización de la interpretación literal. Por lo expuesto, se concluye que nuestra constitución establece que las disposiciones deben ser interpretadas no solo por su tenor literal, sino que además de ello deben ser interpretadas desde la integralidad de la constitución, es decir, observando y analizando a la constitución como un sistema.

La Ley Orgánica de Garantías Jurisdiccionales y Control Constitucional determina que la interpretación sistemática deberá partir del contexto general del texto normativo para así poder lograr una debida coexistencia, correspondencia y armonía entre todas las disposiciones. Bajo el lineamiento de comparar la interpretación literal de la disposición contenida en el artículo 364 de la constitución con el contexto general del texto constitucional y así poder determinar si el resultado interpretativo es una norma constitucional coexistente, correspondiente y armónica con la ley fundamental en su integralidad, procederé a efectuar la referida interpretación.

Entre los artículos 3 y 364 de la constitución se evidencia coexistencia, correspondencia y armonía respecto al carácter garante del Estado en materia de derechos constitucionales. Los derechos constitucionales que se observan protegidos en ambos artículos son el derecho a la salud y alimentación, dado que al ser las adicciones un problema de salud pública, el derecho que se pretende proteger es el derecho a la salud y consecuentemente a la alimentación. De igual forma, otro derecho constitucional protegido es el derecho a la libertad, toda vez que el artículo 364 determina la no criminalización del consumo en ningún caso.

Respecto al artículo 10 de la constitución, se identifica coexistencia, correspondencia y armonía entre este y el artículo 364, debido a que el artículo 10 refiere a la titularidad y goce de las personas en derechos constitucionales, y; el artículo 364 evidencia la titularidad y goce de los consumidores respecto a derechos constitucionales.

La coexistencia, correspondencia y armonía existente entre los artículos 11 y 364 de la constitución, radican en el principio de igualdad y no discriminación por ningún tipo de distinción, que tenga como finalidad menoscabar o anular el reconocimiento, goce o ejercicio de los derechos constitucionales, puesto que la norma del artículo 364 determina la no discriminación 
de los consumidores respecto al resto de ciudadanos, al reconocerle en igualdad la titularidad y goce de derechos constitucionales. De igual manera, en ambos artículos se evidencia la garantía de derechos por parte del Estado.

Las compatibilidades encontradas entre los artículos 66 y 364 de la constitución, reposan en el reconocimiento y garantía del derecho a una vida digna y no discriminación de los ciudadanos, toda vez que al ser declaradas las adicciones como un problema de salud pública y ser el Estado, el responsable respecto a la solución de esta problemática a través de distintos programas, lo que se busca con este accionar es garantizar a los consumidores su derecho a la vida digna asegurándole aspectos primordiales como salud, alimentación, vivienda, educación y trabajo dentro de un marco de igualdad y no discriminación.

La coexistencia, correspondencia y armonía existente entre los artículos 76 y 364 se concentran en el derecho a la libertad y la garantía del estado de inocencia, debido a que el artículo 76 dispone que nadie podrá ser juzgado ni sancionado por el cometimiento de un acto que no esté tipificado como infracción de cualquier naturaleza, en consecuencia, quien se encuentre en esta situación no podrá ser sujeto a sanción, de igual forma, el artículo 364 al establecer la no criminalización del consumo y no vulneración de derechos constitucionales, asegura la libertad e inocencia de los consumidores a pesar de que estos se encuentran en contacto directo y tenencia de sustancias sujetas a fiscalización.

La compatibilidad entre los artículos 159 y 364 de la constitución responden al derecho a la seguridad jurídica de los ciudadanos en general, entendiendo que este derecho se fundamenta en el respeto a la constitución y a la existencia de normas previas, claras y públicas, aplicadas por las autoridades competentes. En este aspecto, se evidencia coexistencia, correspondencia y armonía en relación a la seguridad jurídica de los consumidores, puesto que la no criminalización del consumo y no vulneración de sus derechos constitucionales, son normas previas, claras y públicas, por lo que, los miembros de la policía nacional como autoridades competentes deben actuar en estricta observancia a la constitución y sus normas, es decir, por ningún motivo podrán considerar a la acción de consumir como el cometimiento de un delito y por consecuencia, no podrán privar de libertad ni vulnerar algún otro derecho constitucional de los ciudadanos consumidores de sustancias sujetas a fiscalización pese a que ellos se encuentren en contacto directo o posesión de las mismas.

A pesar de las diferentes concepciones de autores respecto a los derechos fundamentales, el denominador común en ellos se fundamenta en la consecución del buen vivir, a través de la garantía del derecho a la vida digna y el goce efectivo de los demás derechos que emanan de la 114 
ley fundamental, y es así como el artículo 277 de la constitución determina que para poder obtener el buen vivir, el Estado debe garantizar los derechos de las personas, colectividades y la naturaleza, entonces, es en esta disposición donde se observa coexistencia, correspondencia y armonía entre el precitado artículo con el artículo 364, puesto que este determina a las adicciones como un problema de salud pública, la no criminalización del consumo y la no vulneración de los derechos constitucionales de los consumidores con la preponderante finalidad de obtener el anhelado buen vivir.

En el artículo 298 de la constitución se evidencia coexistencia, correspondencia y armonía entre la preasignación presupuestaria a los GAD destinadas al sector de la salud con el artículo 364 que determina al Estado como responsable de combatir la problemática de salud pública de las adicciones mediante desarrollo de programas.

Las compatibilidades que reflejan coexistencia, correspondencia y armonía entre los artículo 340 y 364 de la constitución, se concentran en que la constitución a través del artículo 340 ha determinado un sistema nacional de inclusión y equidad social con la finalidad de garantizar los derechos reconocidos en la constitución, de igual forma, el artículo 364 determina que siendo las adicciones un problema de salud pública, el Estado a través de un desarrollo planificado tiene la obligación de garantizar los derechos constitucionales de los consumidores. Asimismo, las normas que emanan de ambos artículos prevén la garantía de los derechos constitucionales mediante la sujeción de los principios de igualdad y no discriminación.

En el artículo 341 de la constitución se evidencian aspectos fundamentales como: responsabilidad del estado de asegurar a sus habitantes los derechos y principios que se desprenden del texto constitucional; puntualización de los principios de igualdad y no discriminación; consecuentemente a esta particularidad, el constituyente reconoce una atención prioritaria a grupos minoritarios, lo cual responde a su constante exposición a la desigualdad, exclusión, discriminación o violencia; utilización de sistemas especializados a fin de proteger integralmente los referidos derechos y principios. Por lo tanto, el artículo 364 expresa coexistencia, correspondencia y armonía al artículo 341, toda vez que este establece la protección de los principios fundamentales y derechos constitucionales de un grupo minoritario como los ciudadanos consumidores y adictos, a través de programas desarrollados y especializados.

En los artículos 360, 361, 362 y 363 de la constitución, se identifica que el sistema de salud será dirigido por el Estado a través de la autoridad sanitaria nacional. En base a esta función de

115 
dirección, el Estado representado por sus órganos competentes debe desarrollar políticas públicas destinadas al campo de la salud con la finalidad de promover la salud, prevenir y atender integralmente a la familia y comunidad. Entonces, el artículo 364 evidencia una clara compatibilidad, dado que, en él también se establecen disposiciones que señalan al Estado como ente rector de las políticas públicas de salud y responsable del desarrollo de programas especializados para la promoción de la salud, prevención y atención integral de los consumidores.

Por las consideraciones expuestas, al haber utilizado un método sistemático consistente en comparar la interpretación de la disposición contenida en el artículo 364 con nuestro texto constitucional en su integralidad, se demuestra que la norma constitucional proveniente de la interpretación del artículo 364, no evidencia incompatibilidades constitucionales, por lo que, es una norma coexistente, correspondiente y armónica respecto a nuestra Ley Fundamental y contribuye a que la constitución sea observada y analizada como un todo o conjunto indivisible.

Siguiendo el esquema interpretativo establecido por la constitución en su artículo 427, en el presente estudio se ha utilizado en primera instancia, una interpretación al tenor literal de la disposición contenida en el artículo 364, lo que ha permitido analizar a la referida disposición a través del conocimiento y entendimiento específico de los vocablos que constituyen el enunciado, para acto seguido poder interconectarlos y obtener una abstracción estrictamente gramatical. Además, continuando con el esquema interpretativo constitucional, se ha efectuado un examen con enfoque sistemático a la constitución, debido a ello, se ha comparado la disposición del artículo 364 con el texto constitucional de forma transversal, integral y sistemática. Como consecuencia a esta actividad interpretativa se ha demostrado que en el artículo 364 no encierra un escenario de ambigüedad o duda al momento de la interpretación, puesto que el constituyente ha sido claro al momento de plasmar la referida norma en la ley fundamental.

\section{Constitucionalidad de la Resolución Nro. 002-CONSEP-CD-2015}

La resolución Nro. 002-CONSEP-CD-2015 fue emitida por el Consejo Nacional de Control de Sustancias Estupefaciente y Psicotrópicas en el año 2015, de acuerdo a lo establecido por la Disposición Transitoria Décimo Sexta de la Ley Orgánica de la Prevención Integral del Fenómeno Socioeconómico de las Drogas y de Regulación y Control del Uso de Sustancias Catalogadas Sujetas a Fiscalización con la finalidad de establecer la tabla de cantidades de sustancias estupefacientes y psicotrópicas en las escalas mínima, mediana, alta y gran escala para efectos de aplicación de lo establecido por la sección segunda, del capítulo tercero, del título cuarto, del libro primero del Código Orgánico Integral Penal.

\section{6}


Por lo expuesto, se identifica que la referida resolución es un acto administrativo, debido a que fue emitida por una persona jurídica autónoma de derecho público con competencia en la materia, como lo fue el Consejo Nacional de Control de Sustancias Estupefacientes y Psicotrópicas; y que en virtud de que dicha institución ejerció sus atribuciones en todo el territorio nacional, se infiere que el referido acto administrativo posee carácter y efectos generales. Adicionalmente, la presente resolución fue emitida para efectos de aplicación del artículo 220 del Código Orgánico Integral Penal, cuya aplicación abarca a todo el ordenamiento jurídico ecuatoriano. Cabe recalcar que el Consejo Nacional de Control de Sustancias Estupefacientes y Psicotrópicas no existe a la actualidad pero que la resolución 002-CONSEP-CD-2015 aún se encuentra vigente.

Consecuentemente, si se pretende efectuar un análisis de constitucionalidad a la resolución 002-CONSEP-CD-2015, la cual configura un acto administrativo de carácter y efectos generales, se infiere que dicho examen de constitucionalidad deberá orientarse a lo establecido por el artículo 74 de la Ley Orgánica de Garantías Jurisdiccionales y Control Constitucional, esto es, el control abstracto de constitucionalidad, cuya competencia recae en la Corte Constitucional de acuerdo a lo dispuesto por los artículos 75, 98, 136 de la referida ley y al numeral 4 del artículo 436 de la constitución, pero que para efectos de esta investigación, se procederá a efectuar en sus aspectos elementales. La finalidad del control abstracto de constitucionalidad reside en garantizar la unidad y coherencia del ordenamiento jurídico a través de la identificación y eliminación de las incompatibilidades normativas entre las normas constitucionales y las demás disposiciones integrantes del sistema jurídico; dichas incompatibilidades pueden ser por aspectos de fondo $\mathrm{y}$ forma.

En el presente estudio, la disposición acusada como inconstitucional se encuentra en la resolución Nro. 002-CONSEP-CD-2015, específicamente en la parte que determina "Ratificar las tablas de sustancias estupefacientes y psicotrópicas para sancionar el tráfico ilícito de mínima, mediana, alta y gran escala, previsto en el artículo 220 del COIP, aprobadas mediante Resolución No. 001-CONSEP-CD-2015..." (CONSEP, Resolución 002-CONSEP-CD-2015, 2015, pág. 2).

Las disposiciones constitucionales presuntamente infringidas son las contenidas en el artículo 364 de la constitución, cuyo contenido y alcance residen en tres parámetros principales que son la consideración de las adicciones como problema de salud pública, la no criminalización del consumo y la no vulneración de los derechos constitucionales de los consumidores. 
Los argumentos por los cuales se considera que existe una incompatibilidad normativa residen en la discordancia y no coexistencia entre la tabla de sustancias estupefacientes y psicotrópicas contenida en la resolución 002-CONSEP-CD-2015, la cual se encuentra orientada a sancionar el tráfico ilícito de sustancias catalogadas sujetas a fiscalización, específicamente en lo que respecta a la marihuana (cannabis sativa), en los parámetros correspondientes al tráfico a mínima escala; y, la resolución 001-CONSEP-CD-2013, que acoge los estudios toxicológicos, sociológicos y biológicos elaborados por el Ministerio de Salud Pública sobre la tenencia de sustancias estupefacientes y psicotrópicas para su consumos personal, además de aceptar la propuesta de cantidades máximas admisibles de tenencia para el consumo personal planteadas por el referido ministerio, específicamente en lo que relaciona a la marihuana (cannabis sativa), cuya cantidad máxima admisible de tenencia para consumo personal es 10 gramos como peso neto.

La presunta incompatibilidad normativa motiva su acusación en la criminalización del consumo de marihuana (cannabis sativa) y por consecuencia la vulneración de los derechos constitucionales de los consumidores a la misma, en razón de que existen categorías compartidas entre la resolución 002-CONSEP-CD-2015 que sanciona como tráfico ilícito a mínima escala, la tenencia de más de 0 gramos hasta 20 gramos (peso neto) de marihuana (cannabis sativa); y la resolución 001-CONSEP-CD-2013, que determina como cantidad máxima admisible de tenencia de marihuana (cannabis sativa) la cantidad de 10 gramos (peso neto).

Adicionalmente, la acusación también se fundamenta en que la consideración de la licitud de la tenencia de sustancias catalogadas sujetas a fiscalización por parte de la resolución 002CONSEP-CD-2015 se encuentra únicamente enfocada en parámetros cuantitativos respecto a la tenencia, lo que contraviene a la sentencia 7-17-CN/19 expedida por la Corte Constitucional, la cual orienta la consideración de licitud de la tenencia de sustancias catalogadas sujetas a fiscalización en el destino de la referida tenencia, puesto que, si dicha tenencia supera las cantidades máximas establecidas de consumo personal, pero se encuentra destinada al consumo, esta acción no genera responsabilidad penal para quien la cometa: y, son los operadores de justicia quienes deben establecer que la persona en tenencia de sustancias estupefacientes, psicotrópicas o preparados que las contengan, tengan intención de traficar en lugar de consumir. La referida sentencia posee carácter entre partes y para casos análogos.

Por lo tanto, se constata que una persona detenida por un control policial, a la cual se le efectúa un registro y producto de aquello, se identifica la tenencia de sustancias catalogadas sujetas a fiscalización en las cantidades establecidas en las tablas de sustancias estupefacientes

118 
y psicotrópicas de la resolución 002-CONSEP-CD-2015, será arrestada por parte de los agentes policiales, debido a que se encuentra en el cometimiento de una conducta que se adecua al delito de tráfico ilícito de sustancias catalogadas sujetas a fiscalización.

En base a lo expuesto, es necesario puntualizar que la persona detenida por un control policial, no necesariamente es detenida por encontrarse en el consumo de sustancias estupefacientes 0 psicotrópicas en un espacio público ni por el cometimiento de un delito flagrante, por lo que, se identifica que las razones de la detención han sido motivadas por sospechas de los agentes de control e incluso que, la persona detenida puede ser cualquiera en el ejercicio de su libre tránsito por los espacios públicos. Adicionalmente, en estricta observancia de la resolución 002CONSEP-CD-2015, el agente policial no debe constatar ni considerar si la persona detenida se encuentra traficando las sustancias estupefacientes o psicotrópicas en tenencia, sino que únicamente por encontrarse en la tenencia de cantidades establecidas por las tablas de la resolución 002-CONSEP-CD-2015, la persona detenida se encuentra adecuando su conducta al cometimiento del delito de tráfico ilícito de sustancias catalogadas sujetas a fiscalización determinado en el Código Orgánico Integral Penal, en consecuencia, debe ser privada de su libertad y ser puesta a orden de un juez competente.

Bajo estas consideraciones, se infiere que los problemas jurídicos de los que depende la resolución del presente análisis de constitucionalidad que se plantea esta investigación residen en: ¿La aplicación de la resolución 002-CONSEP-CD-2015 criminaliza el consumo de cannabis sativa y consecuentemente vulnera los derechos constitucionales de sus consumidores? y ¿La aplicación de la resolución 002-CONSEP-CD-2015 cumple con el mandato constitucional que considera a las adicciones como un problema de salud pública? Cabe recalcar que, para efectos de la presente investigación, no ha sido vinculante la necesidad de establecer la criminalización del consumo de otras sustancias catalogadas sujetas a fiscalización, ni la vulneración de los derechos constitucionales de los consumidores a las mismas.

Respecto al primer problema jurídico, se ha identificado que la resolución 002-CONSEP-CD2015 enfoca la sanción del tráfico de sustancias catalogadas sujetas a fiscalización únicamente en la consideración cuantitativa de la tenencia valorada en gramos como peso neto.

Las consideraciones cuantitativas de la resolución 002-CONSEP-CD-2015 se desprenden de las tablas de sustancias estupefacientes y psicotrópicas contenidas en dicha resolución. En esta tabla se identifica que la categoría que sanciona el delito de tráfico ilícito de cannabis sativa en mínima escala se encuentra comprendida en los parámetros que inician desde más de 0 gramos 
hasta los 20 gramos. De igual forma, se identifica que la resolución 001-CONSEP-CD-2013 determina que la cantidad máxima admisible de tenencia de cannabis sativa para consumo personal asciende a los 10 gramos.

Las referidas resoluciones se encuentran vigentes en el ordenamiento jurídico ecuatoriano, y así lo ha ratificado la Corte Constitucional, debido a que en sentencia 7-17-CN/19 se determinó la compatibilidad constitucional de la resolución 001-CONSEP-CD-2013 con el artículo 364 de la constitución, en consecuencia, la vigencia de la mencionada resolución dentro del sistema jurídico. En ese sentido, las dos resoluciones en cuestión pueden ser aplicadas dentro del ordenamiento jurídico; pero al momento de su aplicación, dichas resoluciones generan confusión, dado que existen contradicciones entre ellas, específicamente entre la categoría que sanciona el tráfico ilícito de marihuana (cannabis sativa) a mínima escala (resolución 002-CONSEP-CD2015) y la cantidad máxima admisible de tenencia de marihuana (cannabis sativa) para consumo personal (Resolución 001-CONSEP-CD-2013).

La contradicción expuesta en el párrafo precedente, reside en que las resoluciones 001CONSEP-CD-2013 y 002-CONSEP-CD-2015 comparten categorías en lo que refiere a la cuantificación de la tenencia de cannabis sativa. Esto se evidencia, puesto que una persona que se encuentra en la tenencia de una cantidad admisible para el consumo personal de cannabis sativa, esto es, hasta 10 gramos, adquiere la calidad de consumidor de acuerdo a la resolución 001-CONSEP-CD-2013; pero esa misma persona también se encontraría en la tenencia de una cantidad que es sancionada como delito de tráfico ilícito de marihuana (cannabis sativa) a mínima escala, en razón de que dicha categoría comprende las cantidades que inician desde más de 0 gramos hasta los 20 gramos.

En virtud a lo señalado, es claramente identificable que las resoluciones en cuestión comparten categorías respecto a la tenencia de marihuana (cannabis sativa); lo que genera confusión al momento de su aplicación, en cuanto a establecer la diferencia entre un consumidor y un traficante a mínima escala. Dicha situación conlleva a la criminalización del consumo de marihuana (cannabis sativa), lo que consecuentemente vulnera los derechos constitucionales de los consumidores de esta planta.

Respecto al segundo problema jurídico, una vez identificado que las incongruencias entre las resoluciones en cuestión generan la criminalización del consumo de marihuana (cannabis sativa) y vulneran los derechos constitucionales de los consumidores a la misma, se dilucida que un consumidor que padece de adicciones y que es privado de su libertad por la tenencia de las sustancias estupefacientes o psicotrópicas a las cuales es adicto, no será debidamente atendido 120 
como lo determina el artículo 364 de la constitución, puesto que, un centro penitenciario no es una entidad de salud especializada que pueda tratar su adicción y rehabilitación, por lo tanto, no se cumple con el mandato constitucional que establece a las adicciones como un problema de salud pública y se afecta el derecho a la salud de la persona adicta, además de su privación de libertad.

Una vez analizada todas las cuestiones planteadas, los problemas jurídicos y sus resoluciones, se determina que las resoluciones referidas en el presente examen, evidencian compartir categorías entre la cantidad máxima admisible de tenencia de marihuana (cannabis sativa) para consumo personal y las cantidades de tenencia de marihuana (cannabis sativa) sancionadas como tráfico ilícito en mínima escala, lo que genera la criminalización del consumo de cannabis sativa y la vulneración de los derechos constitucionales de sus consumidores. De igual forma, se establece que la criminalización del consumo de cannabis sativa conlleva la privación de la libertad del consumidor, quién en el caso de padecer una adicción, se verá gravemente afectado al ser ingresado en un centro penitenciario, puesto que, este centro no reúne las características de un centro de salud especializado que pueda tratar su adicción y rehabilitarlo, en consecuencia, se vulnera el derecho a la salud del adicto privado de libertad y no se cumple con el mandato constitucional que determina a las adicciones como un problema de salud pública.

Adicionalmente, el enfoque del marco regulatorio de la resolución 002-CONSEP-CD-2015 únicamente se encuentra orientado a establecer la licitud de la tenencia de sustancias catalogadas sujetas a fiscalización, en base a la cuantificación de la tenencia en gramos/peso neto, y no enfoca la licitud de la tenencia en el destino, es decir, si esta tenencia está destinada al consumo o al tráfico, lo que contraviene la sentencia 7-17-CN/19 y permite la criminalización del consumo y la vulneración de los derechos de los consumidores. Por lo tanto, se identifica que la resolución 002-CONSEP-CD-2015 es incompatible al artículo 364 de la constitución de la república del Ecuador.

\section{CONCLUSIONES}

El nexo entre derechos y garantías no responde a una naturaleza empírica sino normativa, por lo que, la inexistencia de garantías dentro del ordenamiento jurídico son lagunas que el poder público se encuentra en la obligación de subsanar. De igual forma, se señala que la garantía de los derechos fundamentales se encuentra intrínsecamente ligada a la democracia, puesto que,

121 
un Estado que cumple con los derechos fundamentales de sus ciudadanos no solo aporta a su bienestar, sino que además fomenta la democracia, la estabilidad política y social.

Bajo esas consideraciones, la aplicación del artículo 364 de la constitución dentro del ordenamiento jurídico, deviene en la aplicación de una garantía normativa, puesto que, este tipo de garantías se encuentran orientadas a resguardar la dignidad humana de los ciudadanos, consecuentemente, el referido artículo establece de manera positiva que en ningún caso el consumo de cannabis sativa podrá ser criminalizado ni se podrán vulnerar los derechos constitucionales a los consumidores de la misma, por lo que, a fin de poder fundamentar lo expresado, esta investigación utilizó el marco interpretativo constitucional determinado en nuestra ley fundamental y diversos principios y métodos interpretativos aportados por la doctrina, lo que demostró que al someter a la disposición contenida en el artículo 364 de la constitución a la actividad interpretativa literal y sistemática, se obtuvo como resultado una norma constitucional que determina la no criminalización del consumo de cannabis sativa en ninguna situación.

El conocimiento de los derechos fundamentales como derechos positivamente válidos, no debe enfocar únicamente su estudio en la descripción del derecho legislado, sino que su estudio también debe abarcar a la praxis judicial a través de la jurisprudencia, lo cual responde a la vaguedad de los derechos fundamentales, puesto que, en diversas ocasiones no se obtiene mucha ganancia del derecho legislado, lo que obliga a que todo operador del derecho deba imperativamente observar, considerar y recurrir a las jurisprudencias emitidas por los tribunales o cortes constitucionales.

En consecuencia, esta investigación ha recurrido a la sentencia 7-17-CN/19 emitida por la Corte Constitucional, la cual ha ratificado la compatibilidad constitucional de la resolución 001CONSEP-CD-2013 con el artículo 364 de la constitución, por lo tanto, se identifica que la referida resolución se encuentra vigente en el ordenamiento jurídico ecuatoriano. En esa línea de análisis, a través del estudio de la resolución 001-CONSEP-CD-2013, se ha determinado como resultado que, la cantidad máxima admisible de tenencia de cannabis sativa para consumo personal es de 10 gramos/peso neto.

Un problema jurídico que surge a partir de los derechos fundamentales, radica en que los textos constitucionales someten al poder legislativo, ejecutivo y judicial bajo las normas de los derechos fundamentales con la consideración de que estos son de vigencia inmediata y que ejercen un control global del ordenamiento jurídico a través de los tribunales o cortes constitucionales. En aquel sentido, se dilucida que todas las normas jurídicas son susceptibles a un control de constitucionalidad, puesto que las mismas deben ser interpretadas desde la 122 
constitución y evidenciar que son coexistentes, correspondientes y armónicas al texto constitucional. Adicionalmente, es imprescindible señalar que, el nuevo paradigma constitucional responde al principio de estricta legalidad o legalidad sustancial, es decir, que la ley y actos normativos ya no se encuentran sujetos únicamente a vínculos formales, sino que se encuentran sujetos a vínculos sustanciales impuestos por los principios y derechos fundamentales contenidos en las constituciones.

Consecuentemente a aquello, esta investigación ha realizado un examen de constitucionalidad a la resolución 002-CONSEP-CD-2015, a través de la aplicación del control abstracto de constitucionalidad. Por lo tanto, a través del referido examen de constitucionalidad se ha obtenido como resultado, que la referida resolución genera la criminalización del consumo de cannabis sativa y la vulneración de derechos constitucionales de los consumidores de la referida planta.

Mediante el desarrollo de la dimensión analítica de la dogmática jurídica se ha identificado que, en el nuevo paradigma constitucional, la estructura del sistema jurídico se encuentra irradiada por los derechos fundamentales en su totalidad. El constitucionalismo ha resultado en la positivización de los derechos fundamentales, por lo que, esta investigación considera que los derechos fundamentales se transforman en derechos constitucionales en el momento que se introducen en los textos constitucionales de los diversos ordenamientos jurídicos.

Por lo tanto, se ha determinado como derechos constitucionales vulnerados por la resolución 002-CONSEP-CD-2015, al derecho a la salud, libertad, no discriminación, seguridad jurídica, igualdad formal y material, sin perjuicio de que otros estudios determinen distintos derechos constitucionales vulnerados a los ya mencionados, puesto que, una característica esencial de los derechos constitucionales reside en su interdependencia.

\section{REFERENCIAS BIBLIOGRÁFICAS}

7-17-CN/19, 7-17-CN (Corte Constitucional del Ecuador 2 de Abril de 2019).

Alexy, R. (1993). Teoría de los Derechos Fundamentales. Madrid: Fareso S.A.

Alva, R. Á., \& Kuri-Morales, P. (2018). Salud Pública. Mexico: El Manual Moderno S.A.

Cano, N. V., \& Llano, J. V. (2016). Derechos Fundamentales: Un debate desde la argumentación jurídica, El Garantismo y El Comunitarismo. Novum Jus. doi:10.14718/NovumJus.2016.10.2.2

123 
CONSEP. (20 de Junio de 2013). Resolucion 001-CONSEP-CD-2013. Quito, Pichincha, Ecuador: Registro OFicial.

CONSEP. (16 de NOVIEMBRE de 2015). Resolución 002-CONSEP-CD-2015. QUITO, PICHINCHA, ECUADOR: REGISTRO OFICIAL.

Descals, A. M. (2014). Comportamiento del Consumidor. Barcelona: UOC.

Ecuador, C. d. (2008). Constitución de la República del Ecuador.

Ferrajoli, L. (2007). Los fundamentos de los derechos fundamentales. Trotta.

Mesa, V. N. (2003). Teoría Constitucional e Instituciones Políticas. Bogotá: Temis S.A.

Nacional, A. (22 de octubre de 2009). Ley Orgánica de Garantías Jurisdiccionales y Control Constitucional. Quito, Pichincha, Ecuador : Registro Oficial.

Nacional, A. (10 de febrero de 2014). Código Orgánico Integral Penal. Quito, Pichincha, Ecuador: Registro Oficial.

Nacional, A. (26 de Octubre de 2015). Ley Orgánica de Prevención Integral Fenómeno Socioeconómico de Drogas. Quito, Pichincha, Ecuador: Rehistro Oficial.

Pazmiño, E. (2016). LA NO CRIMINALIZACIÓN DEL CONSUMO DE DROGAS. DEFENSA Y JUSTICIA, Edición Especial(23), 13-14. Obtenido de www.defensoria.gob.ec

RAE. (2020). Real Academia Española. Obtenido de Real Academia Española: https://dle.rae.es/adicci\%C3\%B3n?m=form 